\title{
Patienten wollen die Online-Verordnung
}

\author{
Ein Online-Kontakt zum Arzt und dann die verschreibungspflichtige \\ Arznei verordnet bekommen: Viele Patienten in Deutschland \\ können sich das laut einer Umfrage vorstellen. Allerdings nicht \\ ganz ohne Einschränkungen.
}

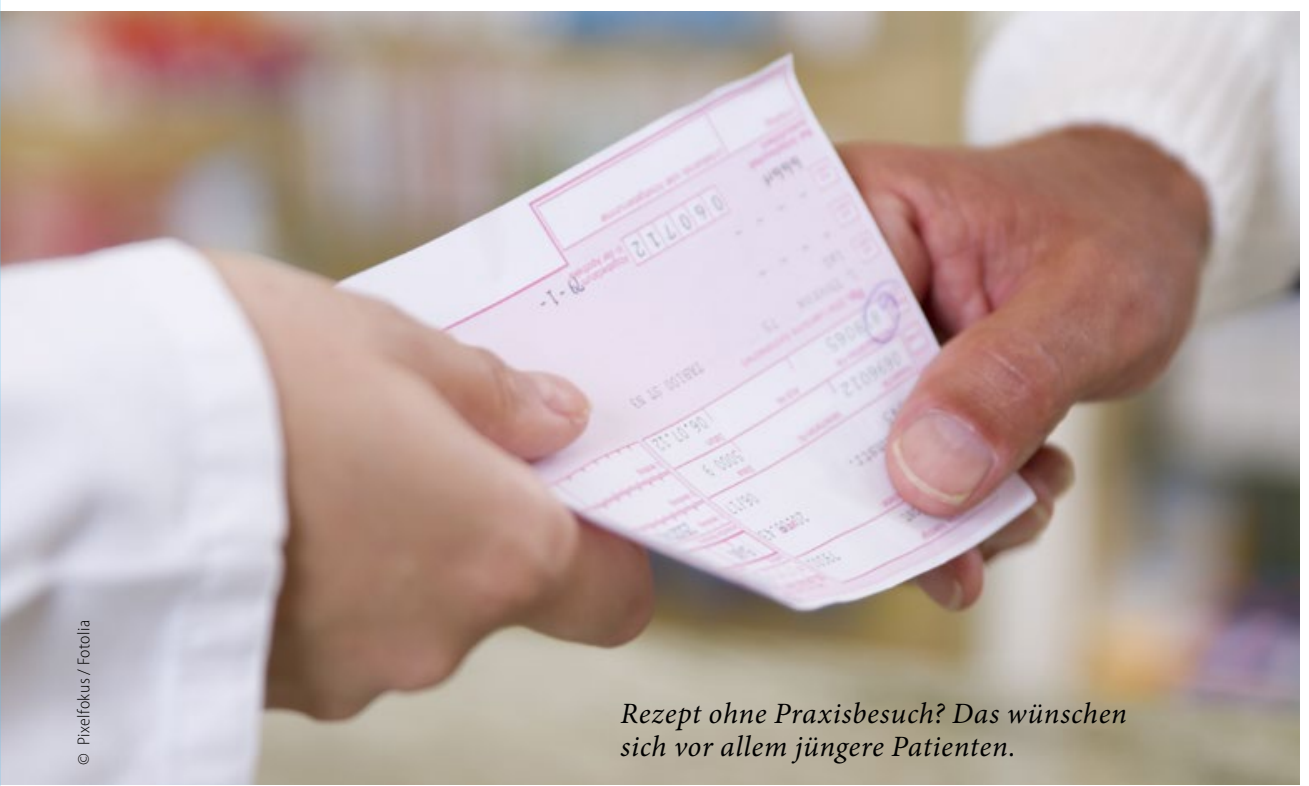

Die Mehrheit der Deutschen wünscht sich von ihrem Arzt Rezepte per OnlineVerbindung oder Telefon. Damit wollen sie vor allem lange Anfahrtswege und Wartezeiten vermeiden, die bei einem Arztbesuch anfallen. Im Zweifelsfall soll der Mediziner entscheiden, ob eine Beratung vor Ort in der Praxis notwendig ist. Das zeigt eine aktuelle Umfrage der Bertelsmann-Stiftung, deren Ergebnisse jetzt veröffentlicht wurden. Für die repräsentative Bevölkerungsbefragung wurden insgesamt 1.005 Bundesbürger ab 14 Jahren zum Thema interviewt.

„Die Umfrageergebnisse lassen erkennen, dass sich die Kommunikationsgewohnheiten der Bevölkerung geändert haben und der Wunsch nach entsprechenden Services in den Arztpraxen wächst", erklärt ein Sprecher der Bertelsmann-Stiftung. 54\% der Befragten wollen den Arzt für die Verschreibung eines Medikaments auch online oder telefonisch konsultieren können - und mit diesem dann klären, ob ein Praxisbesuch wirklich nötig ist. Vor allem Jün- gere (14- bis 29-jährige Umfrageteilnehmer) propagieren so einen Service: $72 \%$ plädieren für eine telemedizinische Verordnungsoption.

\section{Fernverschreibung bisher verboten}

Aktuell sind solche Fernverschreibungen jedoch noch strittig und damit auch der Service, den die Befragten gerne in Anspruch nehmen würden. Der Grund: Nach einer Änderung des Arzneimittelgesetzes, die in Zukunft gelten soll, können Patienten verschreibungspflichtige Medikamente nur dann bekommen, wenn vorher ein direkter Kontakt zum Arzt stattgefunden hat. Damit will der Gesetzgeber die Versorgungsqualität konstant halten. Was das konkret bedeutet, ist noch nicht geklärt. Die Gesetzesnovelle wird derzeit kontrovers in der Öffentlichkeit diskutiert.

In der Umfrage wurde allerdings nicht nur abgefragt, ob Fernverschreibungen grundsätzlich gewünscht werden. Es wurde darüber hinaus zwischen „Erst- verschreibungen“ und „Folgerezepten“ unterschieden.

\section{Zweifel bei Erstverschreibung}

Befragt nach einer telemedizinischen Erstverschreibung fiel das Echo unter den Teilnehmern der Umfrage wesentlich vorsichtiger aus: Lediglich $30 \%$ können sich vorstellen etwa ein Antibiotikum gegen Bronchitis zu erhalten, ohne vorher persönlich vom Arzt beraten worden zu sein. Immerhin $42 \%$ können sind vorstellen, dass die Verschreibung einer rezeptpflichtigen Kortisonsalbe rein per Telefon, Video oder Online-Chat erfolgt. Das deutet darauf hin, dass der Wunsch nach Erstverschreibungen ohne einen persönlichen Arzt-Patienten-Kontakt auch von einem Großteil der Bevölkerung durchaus kritisch gesehen wird.

Anders verhält es sich hingegen, wenn es sich um Folgerezepte handelt. Die Medikamentenverschreibung nach alleinigem telemedizinischen Arztkontakt erhält vor allem dann Zustimmung, wenn es um ein Rezept für die Pille zur Schwangerschaftsverhütung geht (53\%). 50\% der Umfrageteilnehmer gaben zudem an, sich vorstellen zu können, auf diese Weise Blutdruck senkende Medikamente zu erhalten.

In vielen Praxen Deutschlands kann ein Folgerezept bereits per E-Mail angefordert werden.

\section{Bremsschuh für Telemedizin?}

Nach Lesart der Studienautoren sind die Ergebnisse ein klares Signal dafür, dass ein striktes Verbot von Fernverschreibungen den Bedürfnissen der Patienten heute nicht mehr gerecht wird. „Ein pauschales Verbot von Fernverschreibungen nach einer Online- oder telefonischen Arztkonsultation ist weder im Sinne der Patienten, noch ist es sinnvoll im Hinblick auf die Erprobung telemedizinischer Innovationen", heißt es seitens der Bertelsmann-Stiftung. Das wird außerdem nicht nur in der Stiftung so gesehen, sondern auch in den ärztlichen Gremien. So habe die Landesärztekammer BadenWürttemberg diesbezüglich kürzlich ihr Bedauern über die Änderung des Arzneimittelgesetzes sehr deutlich gemacht.

Marco Hübner 